\title{
37 SEGUNDOS (2019). LA ASFIXIA NEONATAL, EL MINUTO DE ORO EN LA REANIMACIÓN Y LA HIPOTERMIA TERAPÉUTICA DESDE EL PRISMA DEL CINE
}

\section{7 seconds (2019). perinatal asphyxia, the golden minute of birth and neonatal therapeutic hypothermia from a movie perspective}

Carlos MORENO ZACONETA, Juliana TRINDADE, Thaiana BELEZA

Hospital Materno Infantil de Brasília (Brasil).

Correo eletrónico: zaconeta@uol.com.br

Recibido: 29 de septiembre de 2020

Aceptado 26 de octubre de 2020

\section{Resumen}

El articulo utiliza la película 37 segundos para llamar la atención en relación a un grave problema social que es la asfixia perinatal. Se aborda su prevención durante el acompañamiento prenatal, la importancia del minuto de oro en reanimación neonatal, la hipotermia como forma de prevención de secuela neurológica y el monitoreo con electroencefalograma para el diagnóstico de convulsiones.

Palabras clave: asfixia perinatal; reanimación neonatal; parálisis cerebral.

\section{Summary}

The article uses the film 37 seconds to draw attention to a serious social problem that is perinatal asphyxia. They talk about prevention during antenatal care, the importance of the golden minute in neonatal resuscitation, hypothermia as a form of prevention of neurological sequelae, and monitoring with an electroencephalogram for the diagnosis of seizures.

Keywords: perinatal asphyxia; neonatal resuscitation; cerebral palsy. 


\section{SEGUNDOS (2019). LA ASFIXIA NEONATAL, EL MINUTO DE ORO EN LA REANIMACIÓN Y LA HIPOTERMIA TERAPÉUTICA DESDE EL PRISMA DEL CINE \\ CARLOS MORENO ZACONETA, JULIANA TRINDADE, THAIANA BELEZA}

37 segundos (2019) de Hikari muestra la vida de una persona con parálisis cerebral, evidenciando las dificultades externas (e internas) que esta condición conlleva. Yuma es una joven japonesa usuaria de silla de ruedas que procura afirmarse en el mercado de trabajo, pero también quiere conocer y disfrutar la vida, incluyendo su sexualidad. Aunque la película muestra una ciudad japonesa actual con mucha accesibilidad para usuarios con necesidades especiales, esta no es la realidad

La asfixia perinatal es la causa más frecuente de parálisis cerebral en todo el mundo. Las medidas destinadas a disminuir la mortalidad y la morbilidad asociadas a la asfixia perinatal incluyen: 1) Medidas de prevención primarias, como atendimiento prenatal precoz y universal, mejora de la salud materna y el reconocimiento y tratamiento oportuno de las enfermedades obstétricas. 2) Reanimación neonatal sistematizada e inmediata. 3) Tratamiento adecuado de los pacientes asfixiados ${ }^{1}$. El presente artículo abordará estos tópicos.

\section{Salud materna, reconocimiento y tratamiento de las enfermedades obstétricas}

La prevención de la asfixia neonatal comienza por el asesoramiento preconcepcional, quiere decir que la mujer que pretende un embarazo (o cualquier mujer en edad fértil) debe ser investigada desde el punto de vista clínico, emocional, nutricional y serológico y ser orientada con relación a reposiciones vitamínicas, vacunas, alimentación, hábitos de vida, uso de alcohol, etc.

El acompañamiento Prenatal debe ser un derecho garantizado para todas las embarazadas, ser precoz y completo pues es sin sombra de duda el mejor momento para orientar, detectar y eventualmente corregir factores que puedan culminar en asfixia perinatal. El médico obstetra podrá identificar factores de riesgo o patologías especificas (obesidad, hipertensión, diabetes, incompatibilidad $\mathrm{rH}$, embarazo múltiple, etc.) que puedan alterar adversamente la evolución del embarazo y podrá aplicar medidas preventivas o terapéuticas. Un acompañamiento prenatal completo presupone mayor grado de seguridad durante el parto.

\section{Reanimación neonatal sistematizada e inmediata}

Uno en cada diez recién nascidos necesita de ayuda para iniciar la respiración efectiva al nascimiento ${ }^{1}$. Cuando nasce un bebé que no es prematuro, el profesional responsable por sus cuidados debe responder dos preguntas: ¿está respirando o llorando? 2) ¿tiene un tono muscular adecuado? Si ambas respuestas son satisfactorias, debe retrasarse el pinzamiento del cordón umbilical, dejar el recién nacido piel con piel con su madre y mantener una evaluación lo menos invasiva posible. Esto debe ocurrir en la gran mayoría de las veces. Si cualquiera de las respuestas no es satisfactoria, el cordón debe ser cortado inmediatamente, el recién nascido debe ser colocado bajo una fuente de calor, posicionar la cabeza en leve extensión, aspirar la vía aérea superior solamente si necesario, secar la piel y reposicionar la cabeza. En ese momento debe evaluarse si el recién nascido está bradicárdico (Figura 1) $)^{2}$.

El nombre de "minuto e oro" se debe a que cuanto mayor la demora en iniciar la reanimación, más difícil esta se torna, elevando así el riesgo de lesión cerebral. La ventilación (respiración) es el procedimiento más importante y efectivo en la reanimación neonatal y debe ser establecido antes de cualquier otro como masaje cardiaco o administración de medicamentos ${ }^{1}$. El riesgo de muerte o morbilidad aumenta en $16 \%$ a cada 30 segundos de demora para iniciar la ventilación hasta el 60 minuto de vida ${ }^{3}$.

El punto alto de la película desde el objetivo de este articulo y que explica el título de la misma ocurre casi al final, cuando Yuma viaja a Tailandia para conocer a su hermana gemela 


\section{SEGUNDOS (2019). LA ASFIXIA NEONATAL, EL MINUTO DE ORO EN LA REANIMACIÓN Y LA HIPOTERMIA TERAPÉUTICA DESDE EL PRISMA DEL CINE \\ CARLOS MORENO ZACONETA, JULIANA TRINDADE, THAIANA BELEZA}

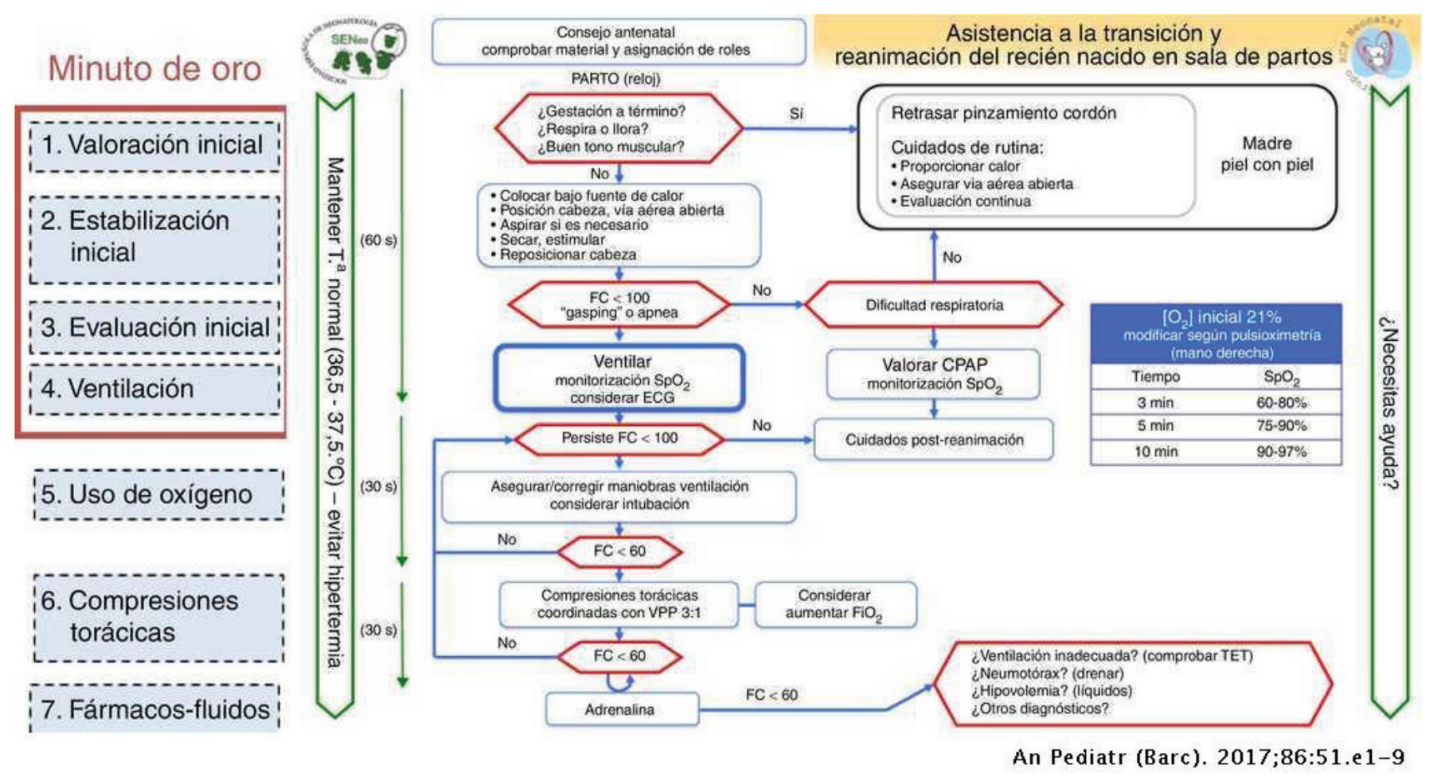

Figura 1. Algoritmo de Reanimación del recién nacido a término. Tomado de An Pediatr (Barc). 2017; 86:51.e1-9

Yuka, que es profesora y lleva una vida normal sin ningún tipo de deficiencia. Ella se desahoga conversando con un amigo y le dice: "fueron 37 segundos que yo demoré para comenzar a respirar", "si yo hubiese respirado un segundo antes, tal vez yo sería libre, igual a ella". Efectivamente, cada segundo vale oro en la reanimación neonatal y por ese motivo el atendimiento tiene que ser inmediato y sistematizado. Antes de cada parto deben estar disponibles para uso todo el material de reanimación, el material humano y deben estar definidas las tareas de cada uno. Esto no se logra improvisando, deben existir cursos regulares de capacitación de los profesionales que atienden en la sala de parto. En este punto es importantísimo recalcar que, en los nacimientos gemelares como es el caso de Yuma e Yuka, debe haber material y equipo profesional de reanimación independiente para cada gemelo ${ }^{1}$.

En la película no se especifica si Yuma nació prematura o no, pero es importante destacar que cuanto menor la edad gestacional, mayor el riesgo de asfixia y mayor la necesidad de reanimación neonatal. Seis en cada 10 recién nacidos con peso inferior a 1500 gramos necesitan de ventilación con presión positiva al nacer ${ }^{1}$.

\section{Tratamiento adecuado de los pacientes asfixiados}

El tercer tópico importante para discutir en este artículo es que cuando la asfixia neonatal es inevitable, debemos impedir de cualquier modo el progreso de la injuria neurológica. El costo económico y social de la parálisis cerebral es tan grande que hoy existe el concepto de UTI Neonatal Neurológica, que son centros altamente especializados, capaces de proveer los más avanzados recursos de monitorización y cuidados cerebrales.

El tratamiento más efectivo para instituir es la hipotermia del recién nascido ${ }^{4}$. El bebé es 


\section{SEGUNDOS (2019). LA ASFIXIA NEONATAL, EL MINUTO DE ORO EN LA REANIMACIÓN Y LA HIPOTERMIA TERAPÉUTICA DESDE EL PRISMA DEL CINE \\ CARLOS MORENO ZACONETA, JULIANA TRINDADE, THAIANA BELEZA}

enfriado a $33,5^{\circ} \mathrm{C}$ durante 72 horas, con lo cual se detiene el proceso de apoptosis neuronal. En la película Yuma tiene 23 años, si hubiera nascido en la última década, ciertamente habría sido sometida a hipotermia y probablemente el grado de secuela neurológica seria mucho menor. Los resultados de ensayos clínicos aleatorios mostraron que la hipotermia redujo significativamente la tasa de muerte y discapacidad importante ${ }^{5}$. Aunque la hipotermia encarece el tratamiento, quedó comprobado que es económicamente más beneficiosa que los gastos totales de niños con secuelas neurológicas ${ }^{6}$.

La monitorización de los signos vitales como presión arterial y oxigenación son muy importantes, pero es crucial la capacidad de diagnosticar o refutar la presencia de convulsiones. Un estudio con 393 casos de convulsión neonatal acompañadas con electroencefalograma (EEG), mostró que $21 \%$ tuvieran signos clínicos mientras $79 \%$ fueron asintomáticos o subclínicos ${ }^{7}$. Por este motivo los recién nacidos asfixiados con encefalopatía deben ser sometidos a EEG continuo o EEG ampliado (EEGa) para no correr el riesgo de dejar de detectar y tratar una convulsión ${ }^{8}$.

Aunque el cine utilizó muchas veces el congelamiento y descongelamiento de personas en innumerables películas de ciencia ficción (Tabla1), la neonatología fue la primera especialidad médica a llevar a cabo con éxito la hipotermia en pacientes humanos (Figura 2).

Concluyendo, la película 37 segundos puede ser utilizada para discutir con estudiantes, profesionales de salud y público en general la importancia del acompañamiento prenatal, el minuto de oro en la reanimación neonatal, la necesidad de un equipo de reanimación para cada gemelo, la hipotermia terapéutica, la UTI neonatal

Tabla 1. El cine utilizó muchas veces la congelación y descongelación humanos como materia de ciencia ficción.

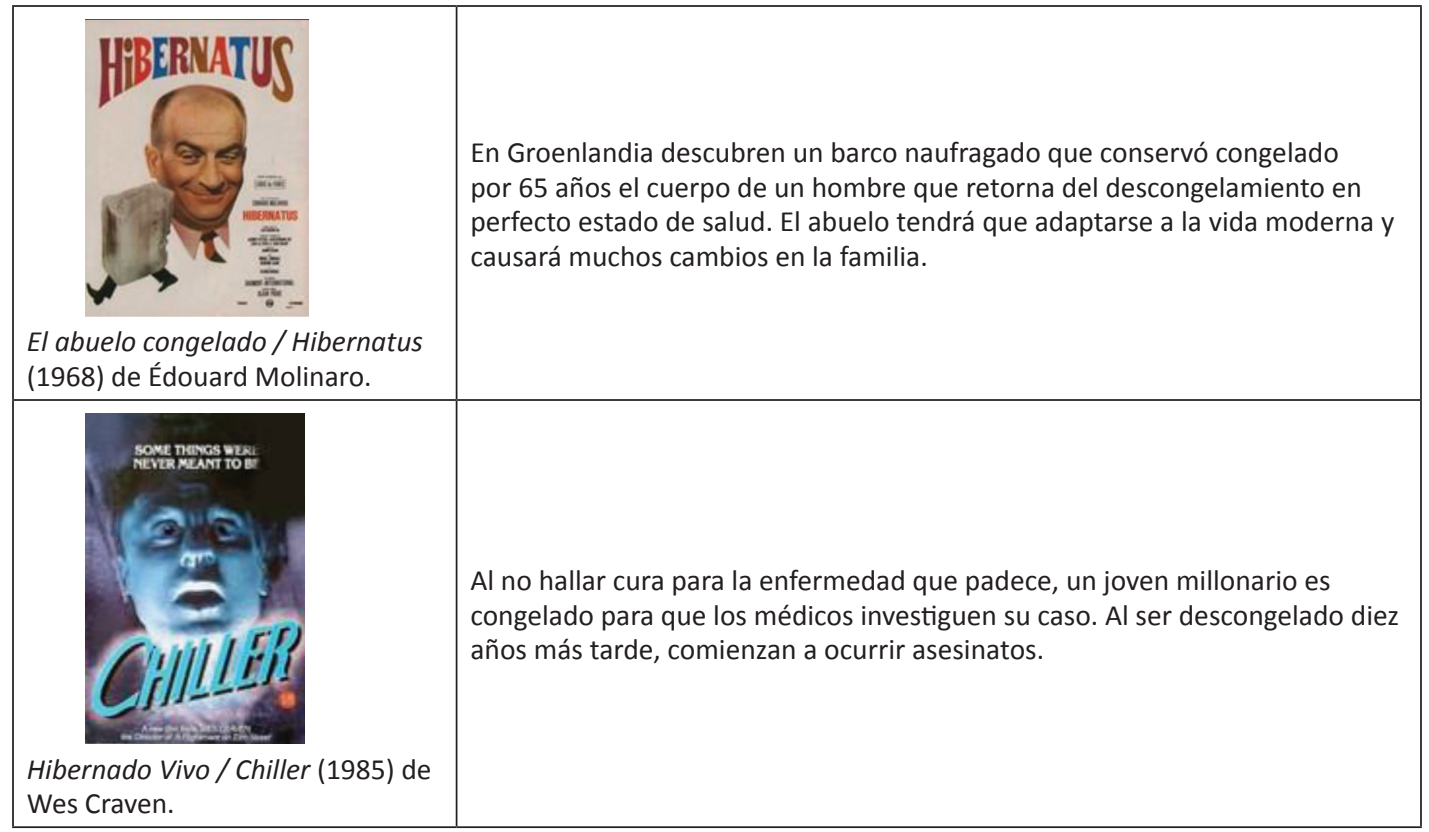




\section{SEGUNDOS (2019). LA ASFIXIA NEONATAL, EL MINUTO DE ORO EN LA REANIMACIÓN Y LA HIPOTERMIA TERAPÉUTICA DESDE EL PRISMA DEL CINE \\ CARLOS MORENO ZACONETA, JULIANA TRINDADE, THAIANA BELEZA}

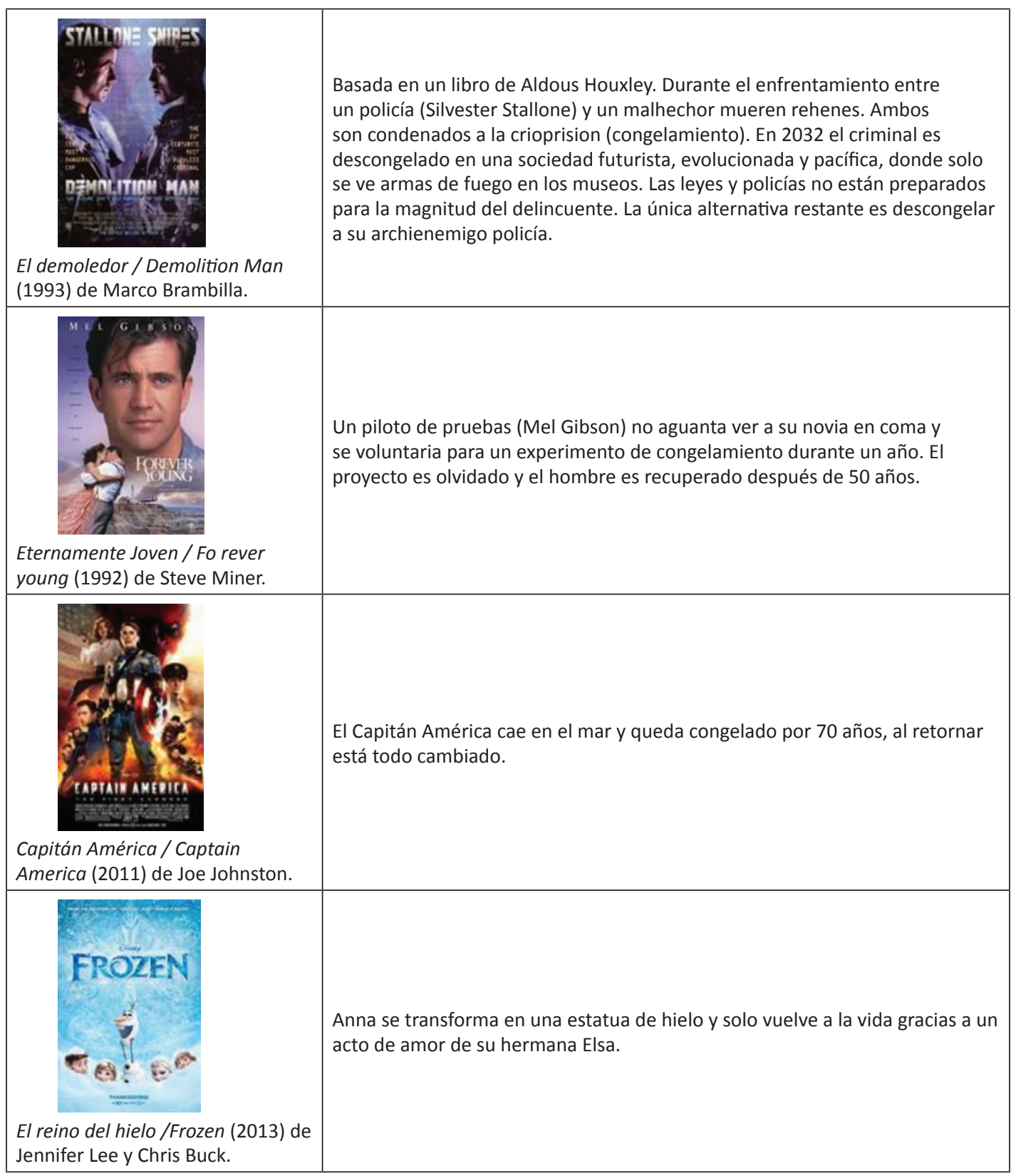

Rev. Med. Cine. 2021; 17 (3), 179-194 Ediciones Universidad de Salamanca / @®@ J. Med. Mov., 2021; 17 (3), $179-194$ 


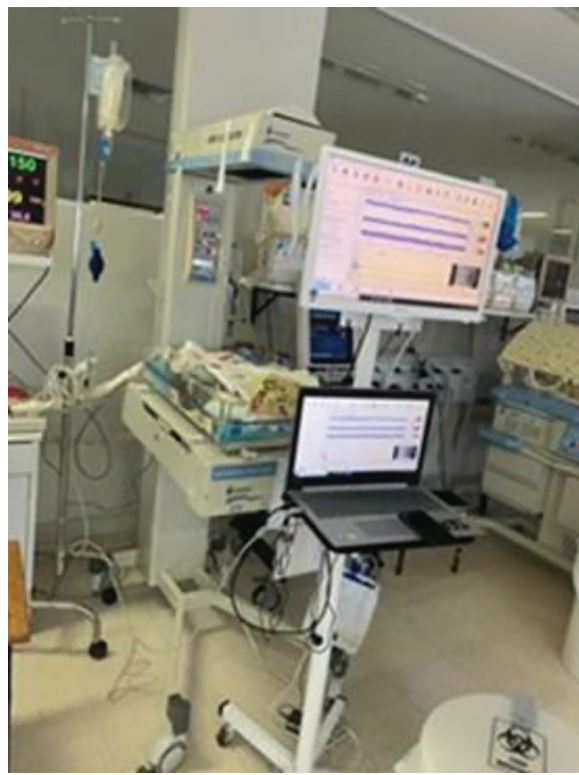

Figura 2. Cuando la realidad supera a la ficción. Recién nacido asfixiado recibiendo hipotermia terapéutica en Brasilia. La lectura continua del EEGa ocurre en otro estado (São Paulo) y en caso de alteraciones se avisa de inmediato por WhatsApp.

neurológica y su relevancia en la disminución de secuelas neurológicas que tanto sobrecargan al individuo, a la familia y a la sociedad.

\section{Ficha técnica}

Título: 37 segundos.

Título original: 37 Sekanzu.

País: Japón.

Año: 2019.

Director: Hikari.

Música: ASKA.

Fotografía: Stephen Blahut.

Montaje: Sawako Ohzu.

Guión: Hikari.

Intérpretes: Mei Kayama, Makiko Watanabe, Misuzu Kanno, Shunsuke daitô, Yuka Itaya,

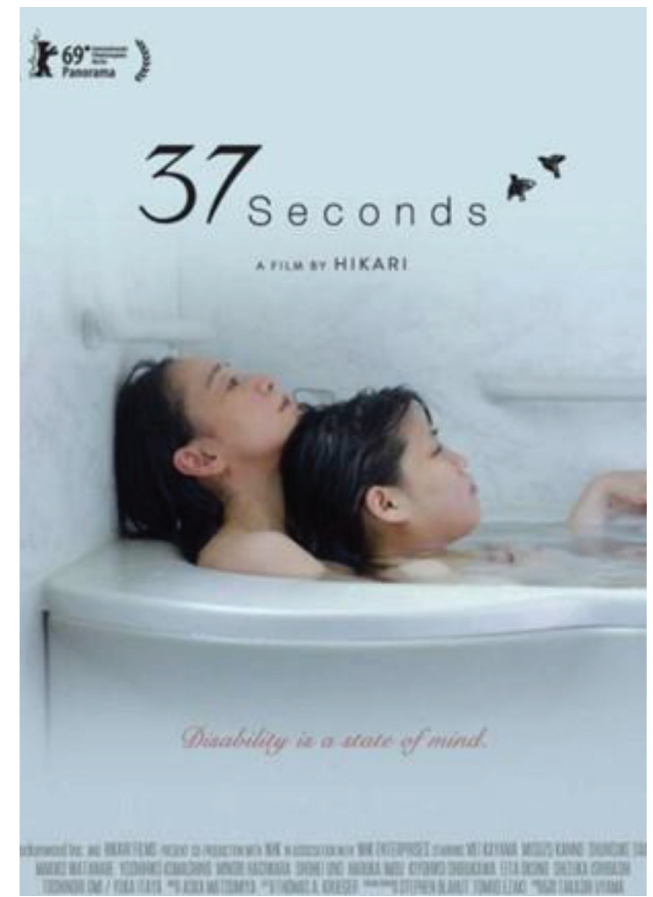

Minori Hagihuara, Shizuka Ishibashi, Kiyohiko Shibukawa, Shohei Uno.

Color: Color.

Duración: 115 minutos.

Género: Drama.

Productores: Hikari, Peter Maestry, Shin Yamaguchi.

Sinopsis: Sofocada por la sociedad y las obligaciones familiares, una artista de manga se embarca en una peculiar aventura en busca de la libertad sexual y la liberación personal.

Premios: Panorama audience award, CICAE art cinema award (2019).

Enlaces: http://37secondsfilm.com

\section{Referencias}

1. Almeida MF, Guinsburg R. Programa de reanimação Neonatal. Manual didático do instrutor. 1th Ed. Programa de reanimação Neonatal. Brasil; 2016. 


\section{SEGUNDOS (2019). LA ASFIXIA NEONATAL, EL MINUTO DE ORO EN LA REANIMACIÓN Y LA HIPOTERMIA TERAPÉUTICA DESDE EL PRISMA DEL CINE \\ CARLOS MORENO ZACONETA, JULIANA TRINDADE, THAIANA BELEZA}

2. Zeballos Sarrato G, Salguero García E, Aguayo Maldonado J, Gómez Robles C, Thió Lluch M, Iriondo Sanz M; Grupo de Reanimación Neonatal de la Sociedad Española de Neonatología (GRN-SENeo). Changes in the international recommendations on neonatal stabilisation and resuscitation (2015). An. Pediatr. (Barc). 2017;86(1):51.e1-51.e9.

3. Ersdal HL, Mduma E, Svensen E, Perlman JM. Early initiation of basic resuscitation interventions including face mask ventilation may reduce birth asphyxia related mortality in low-income countries: a prospective descriptive observational study. Resuscitation. 2012;83(7):869-73.

4. Procianoy R. Hipotermia Terapêutica. Departamento Científico de Neonatologia. Sociedade Brasileira de Pediatria. №4. Junho 2020.
5. Edwards AD, Brocklehurst P, Gunn AJ, Halliday $H$, Juszczak E, Levene $M$, et al. Neurological outcomes at 18 months of age after moderate hypothermia for perinatal hypoxic ischaemic encephalopathy: synthesis and meta-analysis of trial data. BMJ. 2010;340:c363.

6. Regier DA, Petrou S, Henderson J, Eddama O, Patel $\mathrm{N}$, Strohm B, et al. Cost-effectiveness of therapeutic hypothermia to treat neonatal encephalopathy. Value Health. 2010;13(6):695-702.

7. Clancy RR, Legido A, Lewis D. Occult neonatal seizures. Epilepsia. 1988;29(3):256-61.

8. Variane G, Magalhães M. Departamento Científico de Neonatologia. Sociedade Brasileira de Pediatria. №8. Setembro 2020. 


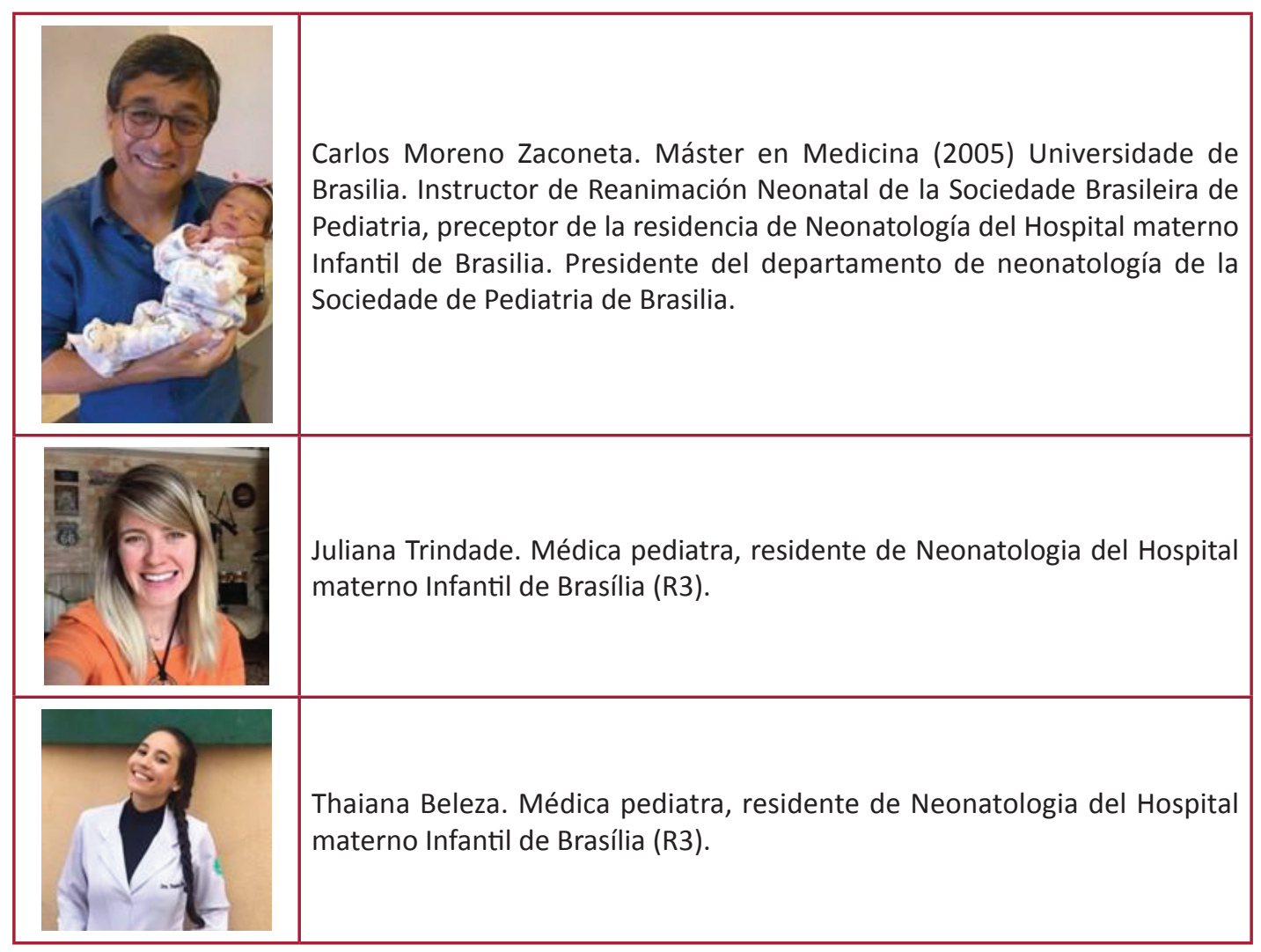

